\title{
O benefício da dança sênior em relação ao equilíbrio e às atividades de vida diárias no idoso
}

\author{
The senior dance benefit in equilibrium and daily living activities in elderly \\ Aline Felipe Gomes da Silva ${ }^{1}$, Andréa Marques Berbel ${ }^{1}$ \\ ${ }^{1}$ Curso de Fisioterapia da Universidade Nove de Julho (UNINOVE) - São Paulo (SP), Brasil.
}

DOI: http://dx.doi.org/10.7322/abcshs.v40i1.698

\section{RESUMO}

Introdução: Com a chegada da velhice, ocorrem mudanças que determinam uma progressiva perda da capacidade de adaptação do indivíduo, resultando em dificuldades para manter o equilíbrio ao realizar suas atividades de vida diárias, dificultando a convivência na sociedade. A dança, por sua vez, proporciona a melhora da capacidade funcional e dos benefícios psicológicos. Objetivo: Avaliar o benefício da dança sênior em relação ao equilíbrio e às atividades de vida diárias no idoso. Métodos: Participaram do grupo de dança sênior 19 idosos entre 60 a 85 anos, que responderam as escalas de equilíbrio de Berg e de atividades de vida diárias de Lawton. A dança sênior foi aplicada duas vezes por semana, durante três meses e, ao final desse período, foram refeitas as duas escalas citadas. Resultados: A partir dos achados deste estudo, foi possível identificar que a prática de dança sênior trouxe melhora do equilíbrio e das atividades de vida diárias. Considerando que o equilíbrio e o risco de queda avaliados pela escala de Berg resultaram em avanços, mostrando um valor estatístico significativo de $\mathrm{p}=0,001$ referente à independência nas atividades de vida diárias avaliadas pela escala de Lawton, observou-se o resultado de aperfeiçoamento com $p=0,001$. Conclusão: $A$ dança sênior demonstrou ser benéfica em relação ao equilíbrio e às atividades de vida diárias no idoso.

Palavras-chave: idoso; dança; equilíbrio; atividades cotidianas.

\begin{abstract}
Introduction: With elderly onset, some changes happen and determine a progressive loss of the individual's adaptability, resulting in difficulties to maintain balance when performing activities of daily living, and making coexistence in society difficult. Dance provides improved functional capacity and psychological benefits. Objective: To evaluate the benefit of senior dance regarding balance and activities of daily living in the elderly. Methods: The senior dance group included 19 aged individuals between 60 to 85 years old, who answered the Berg and Lawton activities of daily living scales. Senior dance was applied twice a week for three months and, at the end of this period, the two scales were reapplied. Results: From the results of this study, it was possible to notice that the senior dance practice provided improvements to balance and activities of daily living. Since balance and fall risks assessed by Berg's scale resulted in advances, showing a highly significant statistical value of $p=0.001$ regarding independence in activities of daily living assessed by Lawton's scale, it was observed an improvement result with $p=0.001$. Conclusion: Senior dancing proved being beneficial as to balance and activities of daily living in the elderly.
\end{abstract}

Keywords: aged; dancing; balance; activities of daily living.

Recebido em: 24/03/2014

Revisado em: 29/09/2014

Aprovado em: 16/01/2015

Autor para correspondência: Aline Felipe Gomes da Silva - Studio 11 Pilates - Rua Salvador Simões, 867 - Alto do Ipiranga - CEP: 04276-000 - São Paulo (SP), 


\section{INTRODUÇÃO}

Sabemos que, com a chegada da velhice, ocorrem mudanças morfológicas, funcionais, bioquímicas e psicológicas que determinam uma progressiva perda da capacidade de adaptação do indivíduo e resultam em dificuldades para o convívio em sociedade. Essas alterações estão fortemente associadas a maior prevalência de distúrbios e agravos não transmissíveis e à redução da capacidade funcional para realização das atividades de vida diária (AVD), que, somadas, podem gerar redução da independência e autonomia do idoso, deixando-os mais vulneráveis e aumentando a incidência de processos patológicos, podendo resultar em óbito ${ }^{1,2}$.

No entanto, a longevidade não deve impedir que o idoso conduza sua própria vida de forma autônoma e decida sobre seus interesses. Esse idoso, que mantém sua independência e autodeterminação, ou seja, a capacidade de um indivíduo poder exercer sua autonomia deve ser considerado saudável, ainda que apresente uma ou mais doenças crônicas ${ }^{3}$.

Segundo a Organização Mundial de Saúde (OMS), a população de idosos aumentará de 8,82 para $13,0 \%$ e este crescimento classificará o Brasil, em 2025, como o sexto país em número de idosos na expectativa de vida, com qualidade. Atualmente, esta é de 60 anos e poderá chegar aos 74 anos em $2025^{4}$.

Para que ocorra este aumento com qualidade, é importante o surgimento de atividades apropriadas para receber e reintegrar essa parte da população, pois o exercício físico no idoso é importante para a manutenção e melhora da disposição, da capacidade física, do estado psíquico e também para impedir ou reduzir a progressão de doenças. Quando um idoso realiza uma atividade, esta proporciona benefícios psíquicos e melhora na sua capacidade física, consequentemente, aprimorando sua qualidade de vida diária ${ }^{5}$.

De acordo com a literatura, uma das modalidades de atividade física que proporciona a melhora da capacidade funcional é a dança, também descrita como atividade física que possibilita uma maior interação com outras pessoas e proporciona benefícios psicológicos ${ }^{6}$.

Segundo Fortes ${ }^{7}$, as danças adaptadas podem ser uma opção de atividade física na terceira idade, sendo desenvolvida em grupo, pois facilita a integração, o fortalecimento de amizades, a superação de limites físicos e a dedicação de tempo para si mesmo, consequentemente reduzindo as angústias, os medos e as inseguranças ${ }^{7}$. Além disso, esta prática também proporciona a manutenção do equilíbrio, diminuindo riscos de quedas, melhorando o condicionamento físico e possibilitando uma maior socialização ${ }^{8}$.

No Brasil, um tipo de dança que está ganhando espaço é a Dança Sênior, considerada como um programa para idosos que teve início em 1974, na Alemanha. A dança é baseada em músicas folclóricas de diversos povos e, na maioria das vezes, são realizadas em roda, revivendo cantigas e cirandas da infância. Enquanto dançam, os participantes, envolvidos pela música, ativam a memória relembrando as coreografias, executando exercícios de lateralidade e movimentos delicados de habilidades ${ }^{7,9}$.
A dança sênior é uma atividade lúdica e motivante, que surge em meio a diversos recursos terapêuticos, usada como estratégia preventiva da inatividade para retardar a senilidade e promover a qualidade de vida entre os idosos. É uma opção de atividade sócio-físico-mental e emocional, sendo feita em grupo de baixo impacto, curta duração e que não requer esforços intensos. Suas coreografias podem ser realizadas em ortostase ou sedestação, proporcionando a manutenção da amplitude de movimento, o aumento da flexibilidade, mobilidade e agilidade ${ }^{10}$.

Esta arte, com constante treino de tais coreografias, possibilita o trabalho da coordenação motora, da capacidade cognitiva e visa conservar a capacidade intelectual, desenvolvendo o equilíbrio e mantendo a concentração presente e focada ${ }^{7}$. Durante a atividade em grupo, a autopercepção é importante e constantemente incentivada nos participantes, para que percebam o quanto evoluem nos movimentos, nos equilíbrios físico e emocional e na coordenação motora ${ }^{7}$.

O diferencial desta dança é que ela pode ser adaptada e realizada sentada, assim possibilitando a socialização dos idosos cadeirantes e de pessoas com limitações de marcha e equilíbrio. As danças sentadas são coreografias em que o grupo, em círculo, com todos sentados em cadeiras, executam movimentos coreográficos leves e lentos ao som de músicas específicas ${ }^{7}$.

Essa prática pode ser utilizada como uma estratégia preventiva da inatividade, resultando na melhora significativa do equilíbrio e, consequentemente, evitando quedas e fraturas, as quais ocorrem em grandes quantidades neste período da vida. Além de retardar a senilidade e promover ao idoso uma melhora na AVD, sempre com a expectativa de fazer com que ele não perca a sua essência de existência, diminuindo os graus de riscos ${ }^{11}$.

Em relação às mudanças musculoesqueléticas e biopsicossociais do indivíduo idoso, é importante olhar tal momento com expectativas e adaptações a uma nova realidade. A fim de ajudá-los nesta nova fase de suas vidas, podemos utilizar a dança sênior como uma forma alternativa de atividade física, com repercussão direta sobre a melhora do equilíbrio e nas realizações das AVDs.

O objetivo deste estudo foi avaliar o benefício da dança sênior em relação ao equilíbrio e às AVDs no idoso.

\section{MÉTODOS}

Estudo prospectivo realizado na Universidade Nove de Julho (UNINOVE), em São Paulo, Brasil, de 02 de maio de 2011 a 29 de junho de 2011 e 08 de agosto a 12 de setembro de 2011.

Participaram do grupo de dança sênior 25 idosos com idades entre 60 e 85 anos, dos gêneros masculino e feminino, frequentadores do Parque da Água Branca. Estes formaram uma amostra de conveniência e estavam cientes de que tratava-se de um estudo com a prática deste exercício e que, neste período, não poderiam realizar outra atividade física, sendo que seis indivíduos não foram incluídos na pesquisa por desistência. Assim, demos continuidade com os que permaneceram e completaram o estudo, sendo submetidos à avaliação. 
Os participantes foram orientados quanto ao estudo e à entrega do Termo de Consentimento Livre e Esclarecido. Após aceitação da pesquisa, por meio de assinatura, todos os idosos preencheram a ficha cadastral, na qual informaram seus dados pessoais e possíveis diagnósticos, os quais foram utilizados como critérios de inclusão e exclusão. Após o cadastro inicial, os sujeitos responderam à Escala de Equilíbrio de Berg, na qual foram posicionados em diferentes posturas: sentado para em pé; em pé sem apoio por dois minutos; sentado sem apoio nas costas com pé no chão; braços cruzados; em pé para sentado; passar de uma cadeira com braço para uma sem; ficar em pé por 10 segundos com olhos fechados; em pé sem apoio com os pés juntos; em pé com os membros superiores a $90^{\circ}$ de flexão de ombro e estender os membros superiores o mais longe possível; em pé para pegar um objeto no chão; olhar para trás por cima dos ombros enquanto fica em pé; girar $360^{\circ}$ em torno de si; em pé posicionando os pés alternadamente no degrau sem apoio; em pé sem apoio com um a frente do outro e permanecer em pé sobre uma perna sem apoio. Os escores variaram de 0 a 56 pontos e, quanto maior, melhor o equilíbrio do sujeito avaliado ${ }^{12}$.

Foi aplicada também a escala de Lawton para AVDs, na qual os idosos responderam sobre: cuidados pessoais (alimentação, vestuário, banho, eliminação fisiológica, medicação, interesse na aparência pessoal); cuidados domésticos (preparação de comidas, arrumação da mesa, reparos e trabalhos domésticos); trabalho e recreação (atividades rotineiras, organização e viagens); compras e dinheiro (compra de comida, uso de dinheiro, administração das finanças); locomoção (transporte público, condução de veículos, mobilidade pela vizinhança e locomoção fora de locais familiares); comunicação (uso do telefone, conversas, compreensão, leitura e escrita) e relações sociais (familiares adultos, crianças e amigos). $\mathrm{O}$ resultado foi expresso por meio de três possibilidades: independência de 0 a 8 pontos, grau de dependência de 8 a 24 pontos e dependência completa acima de 24 pontos.

Para compor o grupo de idosos, os idosos deveriam apresentar idade igual ou superior a 60 anos e não serem praticantes de dança sênior.

Foram utilizados os seguintes critérios de exclusão: indivíduos que apresentavam doenças neurológicas (acidente vascular encefálico, Parkinson, Alzheimer e traumatismo crânio-encefálico) e/ou ortopédicas, as quais interferiam no equilíbrio; algum grau de demência, não sendo capazes de atender à voz de comando; dor em repouso ou que impossibilitavam o movimento baseada na escala visual analógica de dor; doenças cardiovasculares (trombose venosa profunda e aneurisma); obesidade mórbida, com menor tolerância à atividade física; faziam uso de medicamentos como ansiolíticos e antidepressivos e aqueles incapazes de permanecer em posição ortostática sem auxílio de dispositivo.

A dança sênior foi executada em pé e sentada, utilizando-se cadeiras. As aulas ocorriam duas vezes por semana, com duração de uma hora cada. A sessão foi iniciada com Geronto-ativação, uma modalidade da dança sênior utilizada como aquecimento que "desperta" o corpo, por meio de exercícios específicos, prevenindo limitações precoces da velhice. Seu objetivo é a ativação pelo "método de 12 minutos", incluindo sessões que estimulam uma parte do corpo de cada vez e favorecem a memorização da sequência dos exercícios e a sua correta aplicação. A coreografia da dança das boas-vindas foi a primeira, pois tem o objetivo de proporcionar socialização e entrosamento do grupo. O mixer sênior é um ritmo mais agitado, que promove interação entre duplas e proporciona trabalhar o equilíbrio. Em Casatshok, há os movimentos que exigem uma interação entre os membros superiores e os inferiores, além de flexibilidade e alongamento. A dança do moinho é uma coreografia que trabalha com a ponta dos pés, a lateralidade corporal e os movimentos das mãos, ajudando a aprimorar o equilíbrio e os movimentos que imitam as AVDs. A valsa sentada tem um ritmo mais calmo e movimentos mais suaves, o que ajuda o idoso a normalizar o seu metabolismo.

As músicas foram alternadas entre lentas e rápidas e com coreografias de baixo impacto, passos leves e curtos, de movimentos suaves, respeitando sempre os limites de cada participante.

Este estudo foi aprovado pelo Comitê de Ética em Pesquisa da UNINOVE, sob o protocolo 398324, de 16 de fevereiro de 2011.

Os valores avaliados apresentaram distribuição não paramétrica, conforme a aplicação do teste de Shapiro Wilk. Para a comparação dos resultados antes e após o período de intervenção proposto, os dados foram obtidos pelas Escalas de Berg e Lawton, sendo tratados por meio do teste estatístico pareado de Wilcoxon. A significância estatística foi estabelecida em $5 \%(\mathrm{p}<0,05)$. O software estatístico SPSS (Statistical Package for Social Science), versão 15.0, foi utilizado nas análises deste estudo.

\section{RESULTADOS}

A Figura 1 apresenta um valor estatístico extremamente significante, de acordo com a Escala de Berg. Resultado semelhante foi encontrado com a aplicação da escala de Lawton, conforme Figura 2. Na Figura 3, observa-se diminuição no risco de quedas, avaliado antes e depois da prática de dança sênior por meio do resultado da Escala de Equilíbrio de Berg. Foi observado, por meio do resultado da Escala de Lawton, um aumento da independência em relação ao antes e depois da prática de dança sênior (Figura 4).

\section{DISCUSSÃO}

A partir desses achados, foi possível identificar que a prática de dança sênior trouxe melhora do equilíbrio e melhor desempenho nas AVDs.

Vale ressaltar que, ao envelhecer, alguns sistemas orgânicos apresentam um declínio de função, sendo comum associá-lo ao processo de envelhecimento, embora muitas dessas alterações possuam poucos efeitos na realização das necessidades diárias da maioria da população idosa ${ }^{13}$.

A mobilidade e o deslocamento no ambiente são elementos essenciais para que as AVDs sejam realizadas com independência. Para executar tais tarefas, é importante que o idoso tenha equilíbrio, destreza nas mudanças de posição e estabilidade ${ }^{14,15}$. 


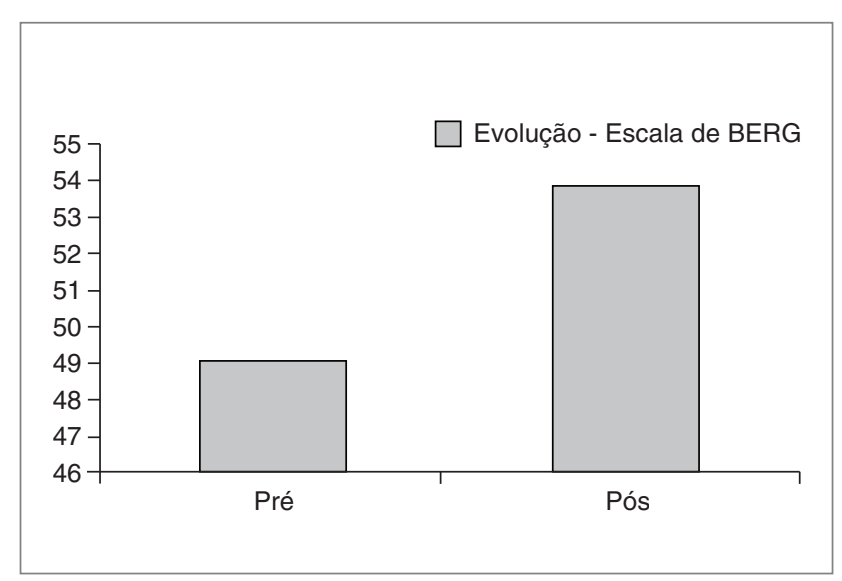

Figura 1: Comparação dos resultados da Escala de Berg (pré- e pós-intervenção), com valor de $p=0,001$

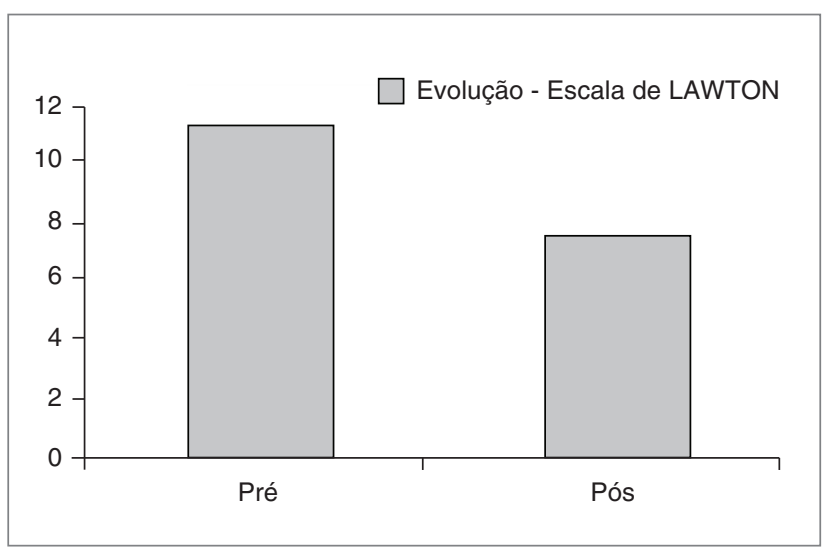

Figura 2: Comparação dos resultados da Escala de Lawton (pré- e pós-intervenção), com valor de $p=0,001$

No presente estudo, utilizamos a dança sênior como uma atividade física, já que, segundo Moreira et al. ${ }^{15}$, "ela requer planejamento, tomada de decisão e monitoramento, tarefas estas relacionadas às funções executivas das AVDs"15.

Na opinião de Ronström ${ }^{16}$, trata-se de um conjunto sistematizado de coreografias baseado em danças folclóricas de diferentes culturas, especialmente adaptadas às possibilidades e necessidades da pessoa idosa ${ }^{16}$.

Como observado, os resultados da Figura 1 referem-se à melhora do equilíbrio nos idosos, podendo ser decorrentes dos movimentos trabalhados nas coreografias, tais como em "Casatschock", que é dançada em pé, na qual o idoso faz apoio unipodal (elevando uma perna de cada vez), associando movimentos de cruzar e bater as mãos, continuando dando três passos para frente e para trás, com inclinação lateral do tronco. Podemos citar também a "Dança do Moinho", na qual o idoso faz movimentos circulares de braço cruzado com o parceiro da dança, associando com movimentos de dorsiflexão e flexão-plantar dos tornozelos, em seguida passos para a lateral sempre no ritmo da dança e associando com os passos do parceiro. Já na coreografia "Mixer Sênior", o idoso realiza quatro passos para trás e para frente, seguidos de um giro para a troca de parceiro.

Em relação aos resultados encontrados sobre a melhora das AVDs, estes puderam ser baseados em movimentos que utilizamos no dia-a-dia, sendo repetidos em algumas coreografias como

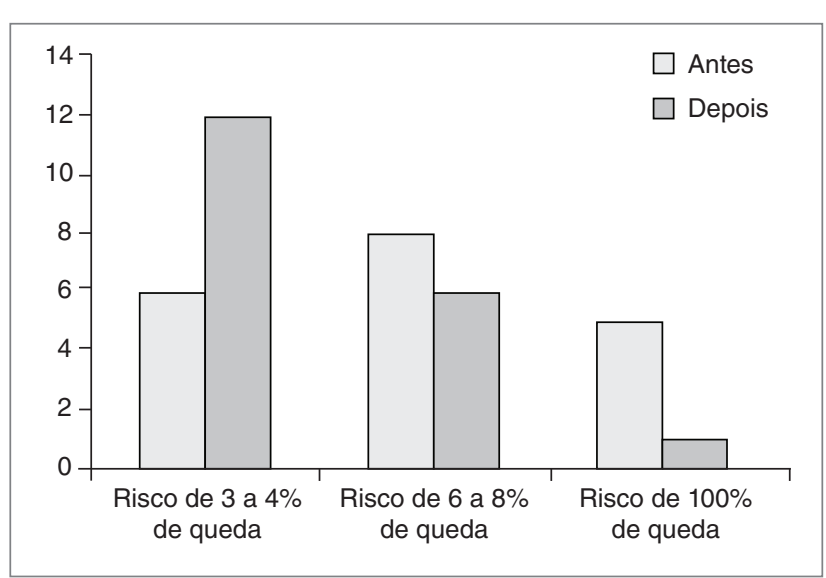

Figura 3: Relação referente ao risco de quedas

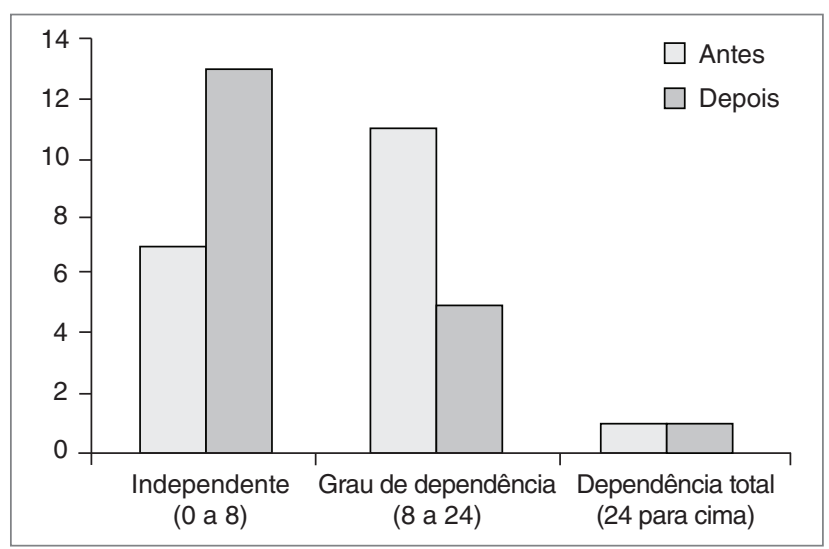

Figura 4: Relação referente à dependência dos idosos

"Boas Vindas", na qual o idoso sentado acaba fazendo uma dissociação da cintura pélvica e leve inclinação do tronco para cumprimentar o parceiro ao lado, além de realizar uma extensão de joelho ativando os músculos quadríceps, finalizando com movimentos dos membros superiores (MMSS) para frente e trás e circulares de baixo para cima. Temos também alguns movimentos na coreografia "Valsa Sentada", na qual o idoso sentado eleva e abaixa os MMSS segurando um lenço, associando com uma inclinação lateral do tronco e finalizando com dois toques de ponta de pé.

Em um estudo realizado por Skelton ${ }^{17}$, o autor defende que a realização da atividade física pode ter efeitos positivos sobre a estabilidade postural e sobre o risco de queda, proporcionando aumento do equilíbrio, da habilidade funcional, da mobilidade, da força e da coordenação ${ }^{17}$.

A Sociedade Brasileira de Geriatria e Gerontologia demonstrou em seus estudos que o risco de quedas relaciona-se com o grau de independência. Assim, idosos que requerem ajuda para a realização das AVDs têm 14 vezes maior probabilidade de caírem, quando comparados aos de mesma faixa etária, porém independentes ${ }^{18}$.

Como demonstrado na Figura 3, o índice de queda diminuiu a partir do momento em que o equilíbrio foi preservado ou adquirido pelos praticantes de dança sênior. Sabe-se que um idoso com episódios de quedas pode desenvolver o que se chama de "síndrome pós-queda", em que o impacto psicológico pode levá-lo à redução das $\mathrm{AVDs}^{19}$. 
De acordo com alguns autores, os idosos são mais propensos a doenças crônicas degenerativas, por exemplo, os processos demenciais, além da perda progressiva de habilidades cognitivas. Uma característica frequente é o declínio da capacidade funcional do indivíduo, que é conceituada como o grau de preservação no desempenho de determinados gestos e $\mathrm{AVDs}^{20,21}$.

Moreira et al. ${ }^{15}$ diferenciam grupos que participavam de programas de atividade física (exercícios aeróbios) e de dança sênior, em que aquele praticante da dança sênior apresentou melhor desempenho, pois esta requer planejamento, tomada de decisão e monitoramento das ações motoras que dependem de coordenação intersujeitos, o que demanda constantes ajustes para que as metas de espaço e tempo sejam alcançadas ${ }^{15,16}$.

Ao contrário dos autores citados anteriormente, Verguese ${ }^{22}$ não encontrou diferenças no desempenho de funções executivas das AVDs entre idosos que praticavam dança e aqueles que a não faziam ${ }^{20}$. Talvez isso tenha ocorrido devido a sua metodologia ter sido em tempo (tanto em horário, como em dias) menor quando comparada com outros estudos, os quais mostraram que a dança era sempre eficaz ${ }^{22}$.

Segundo Brown, Martinez e Parsons ${ }^{23}$, atividades, como caminhar e correr com o objetivo de melhoria cardiorrespiratória, requerem menor participação de funções executivas, quando comparadas às sequenciais que exigem planejamento, tomada de decisão e monitoramento detalhado das ações, funções essas observadas nas atividades coletivas e na dança ${ }^{23}$.

As funções executivas são aquelas utilizadas para desenvolver as AVDs, as quais, no presente estudo, foram avaliadas por meio da escala de Lawton. Esta é um instrumento de rápida e fácil aplicação, que avalia a capacidade de telefonar, viajar, fazer compras, preparar refeições, fazer trabalhos manuais e domésticos, lavar e passar roupas, tomar remédios e cuidar das finanças, na qual seu resultado é expresso por três possibilidades: independência, dependência parcial e dependência completa ${ }^{24}$.

Conforme podemos observar na Figura 4, o resultado deste estudo mostrou que, de 11 idosos com grau de dependência, seis evoluíram para independência. Já dos 7 que eram independentes no início, houve um aumento para 13, dos quais somente um idoso continuou com grau de dependência completa. Entretanto, este mesmo idoso apresentou faltas excessivas no último mês das sessões de dança.

Com relação ao equilíbrio, utilizamos a escala de Berg que está diretamente relacionada a outros testes de equilíbrio e mobilidade, apresentando uma confiabilidade de teste e reteste de $98 \%$. Outra particularidade dessa escala é a relação não linear entre a pontuação e o risco de queda correspondente. Os escores variam de 0 a 56 e, quanto maior, melhor o equilíbrio do sujeito avaliado. Assim, cada ponto a menos na escala corresponde a um aumento do risco de quedas; entre os escores 56 a 54 , cada ponto a menos é associado a um acréscimo de 3 a $4 \%$ no risco de quedas; 54 a 46, a um aumento de 6 a $8 \%$ de chances, sendo que abaixo de 36 pontos, o risco de quedas é de quase $100 \%{ }^{25,26}$.

Quando analisamos a Figura 3 do presente estudo, observamos que houve um decréscimo do risco de queda, mostrando que, antes da prática da dança, cinco idosos tinham 100\% de risco de queda; oito de 6 a $8 \%$; e seis de 3 a $4 \%$. Ao final do período da prática de dança, um participante teve $100 \%$ de risco de queda, que apresentou faltas excessivas no último período da dança, seis idosos com 6 a $8 \%$ do risco de queda e 12 com 3 a $4 \%$ de risco de queda.

Quanto às dificuldades encontradas para a aceitação da prática de dança, pelo sexo masculino, muitos idosos foram convidados a participar, porém apenas quatro deram continuidade, conforme pode ser observado na Tabela 1, portanto ainda existe maior rejeição pelo sexo masculino com relação à pratica de dança.

Diferentemente do sexo feminino que mostra uma aceitação maior, tendo uma desistência mínima por intercorrências como: outros afazeres no mesmo horário, viagem para cuidar da família ou tratamento médico.

Araújo e Alves ${ }^{27}$ destacam que a população brasileira apresenta um maior percentual de mulheres que aceitam e praticam atividade física, tendo consequentemente uma maior expectativa de vida ${ }^{25}$. Esses achados também podem ser observados em nossos resultados, em que o sexo feminino era dominante em relação ao masculino ${ }^{27}$.

É importante ressaltar as limitações encontradas no presente estudo referentes à falta de outras referências bibliográficas relacionadas à prática de dança sênior. Isso vem de encontro com o estudo de Cassiano et al. ${ }^{28}$, os quais encontraram poucas referências nas bases pesquisadas sobre este tema. Tal situação fez com que os autores pesquisassem de forma empírica as mudanças nas esferas sociais, cognitivas, física e emocionais dos participantes, optando por coletarem relatos dos idosos. Sugere-se a realização de mais estudos sobre a dança sênior, com números maiores de participantes, maior tempo de prática $\mathrm{e}$ diferenciação da amostra de grupos. Tais resultados poderão vir de encontro com os expostos no presente estudo, destacando maior confiabilidade à pratica desta dança no idoso e o benefício que este método pode trazer aos seus praticantes.

Tabela 1: Dados referentes ao número, à idade e ao sexo dos participantes que finalizaram a dança

\begin{tabular}{|l|c|c|}
\hline Nome do paciente & Idade & Sexo \\
\hline Idoso 1 & 78 & Feminino \\
\hline Idoso 2 & 62 & Feminino \\
\hline Idoso 3 & 71 & Masculino \\
\hline Idoso 4 & 63 & Feminino \\
\hline Idoso 5 & 82 & Feminino \\
\hline Idoso 6 & 68 & Feminino \\
\hline Idoso 7 & 62 & Feminino \\
\hline Idoso 8 & 60 & Feminino \\
\hline Idoso 9 & 65 & Masculino \\
\hline Idoso 10 & 71 & Feminino \\
\hline Idoso 11 & 62 & Feminino \\
\hline Idoso 12 & 78 & Feminino \\
\hline Idoso 13 & 72 & Feminino \\
\hline Idoso 14 & 80 & Masculino \\
\hline Idoso 15 & 75 & Masculino \\
\hline Idoso 16 & 61 & Feminino \\
\hline Idoso 17 & 60 & Feminino \\
\hline Idoso 18 & 68 & Feminino \\
\hline Idoso 19 & 61 & Feminino \\
\hline
\end{tabular}


Conclui-se que a dança sênior é um método facilitador para trabalhar com o idoso. No presente estudo, houve um resultado benéfico em relação ao equilíbrio e à AVD dos praticantes desta atividade.

\section{AGRADECIMENTOS}

Aos idosos que aceitaram participar deste projeto e à professora Débora Bachin C. de Souza pela colaboração na análise estatística.

\section{REFERÊNCIAS}

1. Figliolino JA, Morais TB, Berbel AM, Corso SD. Análise da influência do exercício físico em idosos com relação a equilíbrio, marcha e atividade de vida diária. Rev Bras Geriatr Geront. 2009;12(2):227-38

2. Freitas EV, Py L Cançado FA, Doll J, Gorzone ML. Tratado de geriatria e gerontologia. 2 ed. Rio de Janeiro: Guanabara Koogan; 2006. p. 846-56.

3. VerasR. Envelhecimento populacional contemporâneo: demandas, desafios e inovação. Rev Saúde Pública. 2009;43(3):548-54. http://dx.doi.org/10.1590/S0034-89102009005000025

4. Amaral JR. Geriatria: principais temas. São Paulo: CBBE; 2009. p. 846-56.

5. Oliveira LC, Pivoto EA, Vianna PC. Análise dos resultados de qualidade de vida em idosos praticantes de dança sênior através do SF-36. Acta Fisitr. 2009;16(3):101-4

6. Gobbi S, Ribeiro CP, Oliveira SR, Quadros Junior AC. Efeitos da dança e do treinamento com pesos nos estados de animo de idosos. Rev Educação Física. 2007;18(2):161-8. http://dx.doi.org/10.4025/reveducfisv18n2p161-168

7. Fortes FM. Superação das limitações da terceira idade através de danças adaptadas. An Prod Academ Docente. 2008; 2(3):419-33

8. Gouvêa JA, Volpato IA, Gilberto AP, Benedeti MR, Bertolini SM, Oliveira AP. Equilíbrio em praticantes de dança sênior em um centro dia do idoso. Rev Científica Jopef. 2013;15(2):24-31.

9. Instituição Bethesda [Internet]. Dança sênior. Disponível em: http://www.portalbethesda.org.br/site_portugues/danca/danca_ senior06.htm. Acesso em: 17 out. 2010.

10. Carvalho PC, Santos LA, Silva SM, Cavalli SS, Corrêa JC, Corrêa FI. Avaliação da qualidade de vida antes e após terapia com dança sênior em pacientes hemiparéticos pós-AVE. Rev Conscientiae Saúde. 2012;11(4):573-9.

11. Carvalho Filho ET, Papeleo Netto M. Geriatria: fundamentos clínica e terapêutica. 2 ed. São Paulo: Atheneu; 2006. p. 24-8.

12. Ribeiro AS, Pereira JS. Melhora do equilíbrio e redução da possibilidade de queda em idosas após os exercícios de cawthorne e cooksey. Rev Bras Otorrnolaringol. 2005;71(1):38-46. http://dx.doi.org/10.1590/S0034-72992005000100008

13. Carvalho Filho ET, Papaleo Netto M, Garcia YM. Biologia e teorias do envelhecimento. São Paulo: Atheneu; 2000. p.3-18.

14. Oliveira DL, Goretti LC, Pereira LS. O desempenho de idosos institucionalizados com alterações cognitivas em atividades de vida diária e mobilidade: estudo piloto. Rev Bras Fisioter. 2006;10(1):91-6.

http://dx.doi.org/10.1590/S1413-35552006000100012
15. Moreira AG, Diniz LF, Fuentes D, Correa H, Lage GM. Atividade física e desempenho em tarefas de funções executivas em idosos saudáveis: dados preliminares. Rev Psiquiatr Clin. 2010;37(3):109-12.

http://dx.doi.org/10.1590/S0101-60832010000300003

16. Ronström O. "I'm old and 'I'm proud". Dance, music and the formation of cultural identity among pensioners in Sweden. Word Music. 1994;3:5-30.

17. Skelton DA. Effects of physical activity on postural stability. Age Ageing. 2001;30(Suppl 4):33-9.

18. Sociedade Brasileira de Geriatria e Gerontologia. Associação Médica Brasileira. Conselho Federal de Medicina. Quedas em idosos: prevenção. Diretrizes. Rio de Janeiro; 2008. p. 1-10.

19. Fabrício SC, Rodrigues RA, Costa Junior ML. Causas e conseqüências de quedas em idosos atendidos em hospital público. Rev Saúde Pública. 2004;38(1):93-9. http://dx.doi.org/10.1590/S0034-89102004000100013

20. Tabert MH, Albert SM, Borukhova-Milov L, Camacho Y, Pelton $\mathrm{G}$, Liu X, et al. Functional deficits in patients with mild cognitive impairment-prediction of AD. Neurology. 2002;58(5):758-64.

21. Rosa TE, Benício MH, Latorre MR, Ramos LR. Fatores determinantes da capacidade funcional entre idosos. Rev Saúde Pública. 2003;37(1):40-8. http://dx.doi.org/10.1590/S0034-89102003000100008

22. Verghese J. Cognitive and mobility profile of older social dancers. J Am Geriatr Soc. 2006;54(8):1241-4. http://dx.doi.org/10.1111/j.1532-5415.2006.00808.x

23. Brown S, Martinez MJ, Parsons LM. The neural basis of human dance. Cereb Cortex. 2005;16(8):1157-67. http://dx.doi.org/10.1093/cercor/bhj057

24. Lawton MP, Brody EM. Assessment of older people: self-maintaining and instrument activities of daily living. Gerontologist. 1969;9(3):179-86.

25. Berg KO, Wood-Dauphinnee SL, Williams JI, Maki B. Measuring balance in the elderly: validation of an instrument. Can J Public Heath. 1992;83(Suppl 2):S7-11.

26. Newstead AH, Hinman MR, Tomberlin JA. Reliability of the Berg balance scale and balance master limits of stability tests for individuals with brain injury. J Neurol Phys Ther. 2005;29(1):18-23.

27. Araújo TC, Alves MI. Perfil da população idosa no Brasil. In: Veras RP, org. Velhice numa perspectiva de futuro saudável. Rio de Janeiro: Universidade do Estado do Rio de Janeiro; Unati; 2001. p. 99-114.

28. Cassiano JG, Serelli LS, Cândido SA, Torquetti A, Fonseca K. Dança sênior: um recurso na intervenção terapêutico-ocupacional junto a idosos hígidos. Rev Bras Cienc Envelhecimento Human. 2009;6(2):204-12.

http://dx.doi.org/10.5335/rbceh.2009.019 